\title{
Superexchange dominates in magnetic topological insulators
}

\author{
Cezary Śliwa, ${ }^{1, *}$ Carmine Autier: (1) 2,3 Jacek A. Majewski, ${ }^{4}$ and Tomasz Diet] [D 2,5, † \\ ${ }^{1}$ Institute of Physics, Polish Academy of Sciences, \\ Aleja Lotnikow 32/46, PL-02668 Warsaw, Poland \\ ${ }^{2}$ International Research Centre MagTop, Institute of Physics, \\ Polish Academy of Sciences, Aleja Lotnikow 32/46, PL-02668 Warsaw, Poland \\ ${ }^{3}$ Consiglio Nazionale delle Ricerche CNR-SPIN, UOS Salerno, I-84084 Fisciano (Salerno), Italy \\ ${ }^{4}$ Institute of Theoretical Physics, Faculty of Physics, \\ University of Warsaw, ul. Pasteura 5, PL-02093 Warsaw, Poland \\ ${ }^{5}$ WPI-Advanced Institute for Materials Research, Tohoku University, Sendai 980-8577, Japan
}

\begin{abstract}
It has been suggested that the enlarged spin susceptibility in topological insulators, described by interband Van Vleck's formalism, accounts for the ferromagnetism of bismuth-antimony topological chalcogenides doped with transition metal impurities. In contrast, earlier studies of $\mathrm{HgTe}$ and related topological systems pointed out that the interband analog of the Ruderman-Kittel-Kasuya-Yosida interaction (the Bloembergen-Rowland mechanism) leads to antiferromagnetic coupling between pairs of localized spins. Here, we critically revisit these two approaches, show their shortcomings, and elucidate why the magnitude of the interband contribution is small even in topological systems. From the proposed theoretical approach and our computational studies of magnetism in Mn-doped $\mathrm{HgTe}$ and CdTe, we conclude that in the absence of band carriers, the superexchange dominates, and its sign depends on the coordination and charge state of magnetic impurities rather than on the topological class of the host material.
\end{abstract}

In the traditional approach to localized magnetism in solids, one considers pairwise exchange interactions between spins $J_{i j}$ comprising the Ruderman-Kittel-KasuyaYosida (RKKY) coupling brought about by band carriers and the Anderson-Goodenough-Kanamori superexchange mediated mainly by anion orbitals [1. However, it has been demonstrated that in the case of p-type dilute magnetic semiconductors (DMSs), the Zener model [2] is remarkably versatile, in which the local magnetization $\mathbf{M}(\mathbf{r})$ plays a role of a continuous order parameter. This approach has allowed understanding the physics of bound magnetic polarons [3, 4] and ferromagnetism of p-type DMSs [5], and subsequently describing quantitatively a wealth of micromagnetic properties and spintronic functionalities of $(\mathrm{Ga}, \mathrm{Mn})$ As and related ferromagnets [6, 7]. Notably, the equivalence between the RKKY and Zener models was established within the mean-field approximation (MFA) 8].

Ferromagnetic topological insulators [9, 10], such as $(\mathrm{Bi}, \mathrm{Sb}, \mathrm{Cr})_{2} \mathrm{Te}_{3}$, have made possible the experimental realization of the quantum anomalous Hall effect [1], the axion insulator [12], efficient magnetization reversal by spin currents [13], and the much disputed chiral Majorana fermions [14, 15]. Interestingly, the appearance of ferromagnetism in these systems is also attributed to their topological character, as the inverted band structure enhances the interband spin susceptibility leading to carrier-independent spin-spin coupling [16], referred to as the Van Vleck magnetism [9, 10, 16]. That appears surprising, however, as early studies of spin-spin coupling mediated by an interband analog of the RKKY interac- tion (the Bloembergen-Rowland (BR) mechanism [17) found predominately antiferromagnetism in topological Mn-doped zero-gap topological HgTe [18 20].

In this Letter, we resolve this puzzle by demonstrating that the mean-field Zener-Van Vleck model fails in the case of magnetism associated with interband bulk excitations in insulators. Furthermore, by making use of the recent progress in the theory of the indirect exchange interaction [21] and in the quantitative description of exchange splitting of bands in the whole Brillouin zone (BZ) 22], we determine various contributions to spin-spin coupling in non-topological CdTe and topological HgTe doped with Mn ions (see Supplemental Material at the manuscript end for an introduction to the topic). We find that the superexchange dominates not only in $(\mathrm{Cd}, \mathrm{Mn}) \mathrm{Te}$ 23, 24, but also in topological $(\mathrm{Hg}, \mathrm{Mn}) \mathrm{Te}$. We also show that the conclusion about the predominant role of the superexchange substantiates the experimental results on $(\mathrm{Cd}, \mathrm{Hg}, \mathrm{Mn}) \mathrm{Te}[25,26$ ] and explains hitherto challenging chemical trends in the magnetic properties of $\mathrm{V}, \mathrm{Cr}, \mathrm{Mn}$, and Fe-doped tetradymite topological insulators observed experimentally [9, 10, 27] and found in $a b$ initio studies [28, 30].

RKKY-BR vs. Zener-Van Vleck models. For concreteness, we consider $x N$ randomly distributed $\mathrm{Mn}$ spins $S=5 / 2$ in zero-gap $\mathrm{Hg}_{1-x} \mathrm{Mn}_{x}$ Te in which both the conduction and valence bands are of $\Gamma_{8}$ symmetry at the $\mathrm{BZ}$ center. In the high-temperature expansion of the partition function for the pairwise interactions 25, the contribution of the RKKY-BR term to the Curie-Weiss temperature (equal to spin ordering temperature $T_{\mathrm{c}}$ within MFA) assumes the form [18, 20, 31, 


$$
\Theta_{\mathrm{CW}}=\frac{x S(S+1)}{3 N \mathcal{V}} \sum_{i \neq j, \mathbf{q}} \exp \left[\mathrm{iq} \cdot\left(\mathbf{R}_{i}-\mathbf{R}_{j}\right)\right] \sum_{\mathbf{k}, n, n^{\prime}, \sigma, \sigma^{\prime}} \frac{2\left|\left\langle u_{n, \mathbf{k}, \sigma}\left|\beta s_{z}\right| u_{n^{\prime}, \mathbf{k}+\mathbf{q}, \sigma^{\prime}}\right\rangle\right|^{2}}{\mathcal{V}\left(E_{n^{\prime}, \mathbf{k}+\mathbf{q}}-E_{n, \mathbf{k}}\right)} f_{n, \mathbf{k}}\left(1-f_{n^{\prime}, \mathbf{k}+\mathbf{q}}\right)
$$

where the Boltzmann constant $k_{\mathrm{B}}=1 ; \mathcal{V}$ is the crystal volume, and $\mathbf{k}, \mathbf{k}+\mathbf{q} \in \mathrm{BZ}$. We see that if the $p-d$ exchange integral $\beta$ were $\mathbf{k}$-independent, the summation over $\mathbf{k}$ would provide the spin susceptibility $\tilde{\chi}(\mathbf{q})$ of the $\Gamma_{8}$ bands $n$ and $n^{\prime}$, as defined in Refs. 32 and 33 . Furthermore, if the contribution of the self-interaction energy were small compared to interaction energies for $i \neq j$, the term $i=j$ could be included in Eq.1. transferring the sum over the cation positions $\mathbf{R}_{i}$ and $\mathbf{R}_{j}$ into the structure factor that is non-zero for $\mathbf{q}=0$ only. This is the case of the long-range RKKY coupling, for which the sum over $i, j$ can be approximated by $N^{2} \beta^{2} \tilde{\chi}(0)$, as presumed within the Zener-Van Vleck model [16, 32, 33. However, in the case of the BR mechanism, the decay of the interaction with the inter-spin distance is faster [17-20] and, therefore, $\tilde{\chi}(q)$ beyond $q=0$ determines the sign and magnitude of $\Theta_{\mathrm{CW}}$ and $T_{\mathrm{C}}$. In conclusion, atomistic computations of pair exchange energies $J_{i j}$ are necessary in order to meaningfully evaluate the role of the interband contribution [34].

Theoretical methodology. We consider exchange interactions between Anderson magnetic impurities occupying cation substitutional positions in considered semiconductor compounds, whose band structures are described within the empirical tight-binding approximation taking into account spin-orbit interactions. This approach [35, 36, 38, was successfully applied to elucidate the nature of ferromagnetism in $(\mathrm{Ga}, \mathrm{Mn}) \mathrm{N}$ [39, 40] and has recently been generalized by us to simultaneously take into account various contributions to the spin pair exchange energy [21, including the BR interband term. Assuming time-reversal symmetry (no spontaneous magnetization) and within the fourth order perturbation theory in the $p$ - $d$ hybridization energy $V_{\text {hyb }}$ between band states $E_{\mathbf{k}, n}$ and Mn $d$ orbitals residing at $E_{d}=E\left(d^{5}\right)-E\left(d^{4}\right)$ and $E_{d}+U=E\left(d^{6}\right)-E\left(d^{5}\right)$, the spin Hamiltonian is,

$$
\hat{\mathcal{H}}_{\mathrm{eff}}^{(4)}=-\sum_{i \neq j} J_{i j, \alpha \beta}^{(4)} \hat{S}_{i, \alpha} \hat{S}_{j, \beta},
$$

where the tensor of exchange integrals (parameters) for spins at sites $(i, j)$ can be written as a double integral over the $\mathrm{BZ}[k \equiv(\mathbf{k}, n)]$ :

$$
J_{i j, \alpha \beta}^{(4)}=-\frac{1}{2(2 S)^{2}} \sum_{m, m^{\prime}} \sum_{k, k^{\prime}} A_{k k^{\prime}}^{(4)} W_{i \alpha, k^{\prime} k, m} W_{j \beta, k k^{\prime}, m^{\prime}}
$$

where $m$ labels the $d$ orbitals and

$$
\begin{aligned}
& W_{i \alpha, k^{\prime} k, m}= \\
& =\sum_{a, b=\uparrow, \downarrow}\left\langle k^{\prime}\left|V_{\mathrm{hyb}}^{\dagger}\right| d_{i} m a\right\rangle\left\langle a\left|\sigma_{\alpha}\right| b\right\rangle\left\langle d_{i} m b\left|V_{\mathrm{hyb}}\right| k\right\rangle,
\end{aligned}
$$

where $a$ and $b$ are spin directions and $\sigma_{\alpha}$ the Pauli matrices. For an insulator in a zero-temperature approximation and using the notation,

$$
\begin{gathered}
w_{k}=\frac{1}{E_{d}+U-E_{k}} ; \quad w_{k}^{\prime}=\frac{1}{E_{d}-E_{k}}, \\
w_{k^{\prime}}=\frac{1}{E_{d}+U-E_{k^{\prime}}} ; \quad w_{k^{\prime}}^{\prime}=\frac{1}{E_{d}-E_{k^{\prime}}},
\end{gathered}
$$

one can write $A_{k k^{\prime}}^{(4)}$ in terms of the Heaviside step function $\Theta$ as,

$$
\begin{aligned}
A_{k k^{\prime}}^{(4)} \approx & \Theta\left(E_{F}-E_{k}\right) \Theta\left(E_{F}-E_{k^{\prime}}\right) w_{k} w_{k^{\prime}}\left(w_{k}+w_{k^{\prime}}\right)+\Theta\left(E_{F}-E_{k}\right) \Theta\left(E_{k^{\prime}}-E_{F}\right) \frac{\left(w_{k}-w_{k^{\prime}}^{\prime}\right)^{2}}{E_{k}-E_{k^{\prime}}} \\
+ & \Theta\left(E_{k}-E_{F}\right) \Theta\left(E_{F}-E_{k^{\prime}}\right) \frac{\left(w_{k^{\prime}}-w_{k}^{\prime}\right)^{2}}{E_{k^{\prime}}-E_{k}}-\Theta\left(E_{k}-E_{F}\right) \Theta\left(E_{k^{\prime}}-E_{F}\right) w_{k}^{\prime} w_{k^{\prime}}^{\prime}\left(w_{k}^{\prime}+w_{k^{\prime}}^{\prime}\right) \\
& +\frac{2}{U}\left[\Theta\left(E_{F}-E_{k}\right) w_{k}+\Theta\left(E_{k}-E_{F}\right) w_{k}^{\prime}\right]\left[\Theta\left(E_{F}-E_{k^{\prime}}\right) w_{k^{\prime}}+\Theta\left(E_{k^{\prime}}-E_{F}\right) w_{k^{\prime}}^{\prime}\right]
\end{aligned}
$$

where we assume that the Fermi energy $E_{F}$ lies in the range $E_{d}<E_{F}<E_{d}+U$. Average values of the exchange integrals and $\Theta_{0}=\Theta_{\mathrm{CW}} / x$ are obtained by tracing the tensors,

$$
J_{i j}^{(4)}=\frac{1}{3} \sum_{\alpha} J_{i j, \alpha \alpha}^{(4)} ; \quad \Theta_{0}=\frac{2}{3} S(S+1) \sum_{i \geq 1} z_{i} J_{0 i}^{(4)}
$$

where $z_{i}$ is a number of cation sites in the consecutive coordination spheres $i \geq 1$.

We represent $A_{k k^{\prime}}^{(4)}$ as a sum of three contributions [23, 41]: the superexchange (or $h h$ ) term includes contributions proportional to $\Theta\left(E_{F}-E_{k}\right) \Theta\left(E_{F}-E_{k^{\prime}}\right)$, the two-electron (or ee) term includes those proportional to $\Theta\left(E_{k}-E_{F}\right) \Theta\left(E_{k^{\prime}}-E_{F}\right)$, and the electron-hole (he) includes those proportional to $\Theta\left(E_{F}-E_{k}\right) \Theta\left(E_{k^{\prime}}-E_{F}\right)$ or $\Theta\left(E_{k}-E_{F}\right) \Theta\left(E_{F}-E_{k^{\prime}}\right)$. Such a decomposition 


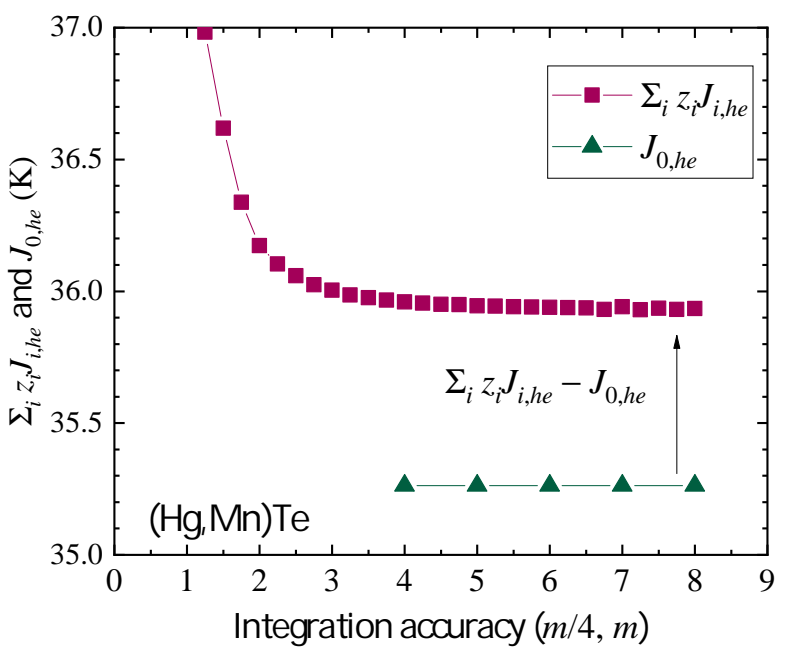

FIG. 1: Convergence of the interband he term for Mn pairs in $\mathrm{HgTe}$ with respect to the number $m$ of $\vartheta$-points in each space direction in the trapezoids quadrature: squares - the sum of exchange integrals including $J_{0}$ calculated for the $4 \times 4 \times 4$ supercell as a single BZ integral (abscissa provides $m / 4$ ); triangles - the value of $J_{0}$ to be subtracted in order to obtain $\Theta_{\mathrm{CW}}$ (abscissa provides $m$ ).

leads to the analogous decomposition of $J_{i j}^{(4)}$ and $\Theta_{0}=$ $\Theta_{h h}+\Theta_{e e}+\Theta_{h e}$.

We use a 16-orbital $s p^{3}$ tight-binding model of the band structure together with the parameter values obtained recently by us employing a modified GGA $+\mathrm{U} a b$ initio approach with $U_{\mathrm{Mn}}=5 \mathrm{eV}$. These parameters are presented in Tables II and III of Ref. 22. In particular, $E_{d}$ in our Eqs. 557 is the mean value of $E_{e g \uparrow}$ and $E_{t 2 g \uparrow}$, whereas $E_{d}+U$ is the mean values of $E_{e g \downarrow}$ and $E_{t 2 g \downarrow}$. Finally, matrix elements of $V_{\text {hyb }}$ are spin-averaged values of $V_{s d} \sigma, V_{p d} \sigma$, and $V_{p d} \pi$ [22]. Extensive magnetooptical data collected for $\mathrm{Cd}_{1-x} \mathrm{Mn}_{x}$ Te and $\mathrm{Hg}_{1-x} \mathrm{Mn}_{x} \mathrm{Te}$ at the $\Gamma$ and $L$ points of the BZ served to benchmark the model [22]. Importantly, our tight-binding model reconfirms for these compounds stronger hybridization of $t_{2 g}$ orbitals with the band states, compared to the $e_{g}$ case, which is crucial for the sign and magnitude of the interaction between localized spins.

In topological materials, the most interesting is the he term. It appears whenever transitions between the fully occupied valence bands and the empty conduction bands are symmetry-allowed (the BR mechanism), or when there is a non-zero density of states at the Fermi level (the RKKY mechanism). This term features an energetic denominator $E_{k}-E_{k^{\prime}}$. In insulators, the latter is guaranteed to be non-zero by the Heaviside- $\Theta$ prefactors (it is understood that each term vanishes whenever the zero Heaviside $-\Theta$ prefactor does, even despite singular denominators). However, in a semimetal, the denominator is singular at the Fermi level (i.e., when either $E_{k} \rightarrow E_{F}^{+}$and $E_{k^{\prime}} \rightarrow E_{F}^{-}$, or vice versa). In undoped (or isoelectronically doped) $\mathrm{HgTe}$, this happens at the $\Gamma$ point of the BZ. For this reason, it has been essential to elaborate a special integration method. The Supplemental Material at the manuscript end presents issues associated with $k$ and $k^{\prime}$ integrations in simple models [18 20 and a comparison of the second-order perturbation theory in the $p-d$ exchange integral compared to the fourthorder perturbation theory in the hybridization matrix element $V_{\text {hyb }}$ employed here.

Brillouin-zone integration. Although our goal is to find the exchange integrals $J_{i j}^{(4)}$ in the limit of an infinitely large system, it is typical in numerical calculations to replace the BZ integration by a summation over a discrete set of $\mathbf{k}$-points in the BZ. As pointed out in Ref. 42, such discrete $\mathbf{k}$-point mesh may be defined through the introduction of the superlattice vectors $\left\{\mathbf{g}_{i}\right\}$, being the linear combinations of primitive crystal translations $\left\{\mathbf{a}_{i}\right\}$,

$$
\mathbf{g}_{i}=\sum_{j=1}^{3} \mathbf{a}_{j} M_{j i} .
$$

In cubic systems the three $\mathbf{g}_{i}$ can be taken as vectors along the three Cartesian axes with length $L a$. This defines the equidistant $\mathbf{k}$-point mesh $\kappa_{\{m\}}=$ $\frac{2 \pi}{L a}\left(m_{x}, m_{y}, m_{z}\right)$ of $L^{3}$ grid points. In addition, the grid points can be shifted.

However, the expressions for the $J_{i j}$ tensor components involve double integration over the BZ. Here, we present an efficient method to deal with the double BZ integration facilitated by the specifics of the integrand. In particular, this method allows for accurate treatment in the he contribution for $J_{i j}$ in the case of zero-gap systems. Indeed, the product $W_{i \alpha, k^{\prime} k, m} W_{j \beta, k k^{\prime}, m^{\prime}}$ includes a phase factor $\exp \left[\mathrm{i}\left(\kappa-\kappa^{\prime}\right)\left(R_{i}-R_{j}\right)\right]$, and the summation over the images of $R_{i}, R_{i}+L a_{\text {lat }}\left(n_{x}, n_{y}, n_{z}\right)$ yields (by the principle of the Poisson summation) a set of Dirac deltas at $\kappa-\kappa^{\prime}=\frac{2 \pi}{L a_{\mathrm{lat}}}\left(m_{x}, m_{y}, m_{z}\right)$,

$$
\begin{aligned}
& \sum_{n_{x}, n_{y}, n_{z}} \exp \left\{\mathrm{i}\left(\kappa-\kappa^{\prime}\right)\left[R_{i}-R_{j}+L a_{\mathrm{lat}}\left(n_{x}, n_{y}, n_{z}\right)\right]\right\}= \\
& =\left(\frac{2 \pi}{L a_{\mathrm{lat}}}\right)^{3} \exp \left[\mathrm{i}\left(\kappa-\kappa^{\prime}\right)\left(R_{i}-R_{j}\right)\right] \sum_{m_{x}, m_{y}, m_{z}} \delta\left(\kappa-\kappa^{\prime}+\frac{2 \pi}{L a_{\mathrm{lat}}}\left(m_{x}, m_{y}, m_{z}\right)\right),
\end{aligned}
$$

where the delta suppresses only one integration. To handle this issue, we first sum over a shifted grid of equidistantly 
spaced $k$-points,

$$
\kappa=\frac{2 \pi}{L a_{\mathrm{lat}}}\left(m_{x}+\frac{\vartheta_{x}}{2 \pi}, m_{y}+\frac{\vartheta_{y}}{2 \pi}, m_{z}+\frac{\vartheta_{z}}{2 \pi}\right) ; \quad \kappa^{\prime}=\frac{2 \pi}{L a_{\mathrm{lat}}}\left(m_{x}^{\prime}+\frac{\vartheta_{x}}{2 \pi}, m_{y}^{\prime}+\frac{\vartheta_{y}}{2 \pi}, m_{z}^{\prime}+\frac{\vartheta_{z}}{2 \pi}\right),
$$

then integrate over the common shift $\left(\vartheta_{x}, \vartheta_{y}, \vartheta_{z}\right) \in[0,2 \pi)^{3} \equiv T^{3}$ ( $T^{3}$ stands for the three-dimensional torus).

In order to calculate $J_{0}+\sum_{i \geq 1} z_{i} J_{i}$ we rewrite 10 with $L=1$ as

$$
\sum_{j} \exp \left[\mathrm{i}\left(\kappa-\kappa^{\prime}\right)\left(R_{i}-R_{j}\right)\right]=\left(\frac{2 \pi}{a_{\text {lat }}}\right)^{3} \sum_{m_{x}, m_{y}, m_{z}} \delta\left(\kappa-\kappa^{\prime}+\frac{2 \pi}{a_{\text {lat }}}\left(m_{x}, m_{y}, m_{z}\right)\right) .
$$

Since $2 \pi / a_{\text {lat }}$ is the lattice constant of the reciprocal lattice, only the term with $m_{x}=m_{y}=m_{z}=0$ remains in the last sum, and the Dirac delta suppresses one integration. Therefore, $J_{0}+\sum_{i \geq 1} z_{i} J_{i}$ and, thus, $\tilde{\chi}(0)$ can be computed as a single integral over the BZ.

In insulators, the integration over $\vartheta$ 's is approximated by a sum over an equally spaced grid (the trapezoids method). The number of required $\vartheta$-points in each Cartesian direction gets smaller as the larger supercells are considered. Ultimately, just one $\vartheta$-point is sufficient; it can be chosen as, e.g., $\vartheta_{x}=\vartheta_{y}=\vartheta_{z}=0$ or $\vartheta_{x}=\vartheta_{y}=\vartheta_{z}=\pi$, in correspondence with the boundary conditions imposed on the electronic wavefunctions. In contrast, in semimetals, if the boundary conditions dictate $\vartheta=0$, finite summation is not appropriate because the denominator $E_{k}-E_{k^{\prime}}$ in Eq. 7 renders the quantity undefined. Therefore, a special set of $\vartheta$-points has been chosen here, which is equivalent to the transformation $\vartheta_{i}^{\prime} \mapsto \vartheta_{i}=\vartheta_{i}^{\prime}-\sin \vartheta_{i}^{\prime}$ under the BZ integral. This transformation, besides possessing analytic properties, preserves periodicity while $d \vartheta_{i} / d \vartheta_{i}^{\prime}=0$ at $\vartheta_{i}^{\prime}=0$. As a result, the divergence of the integral at the origin cancels with the zero of the Jacobian of the transformation and the integral can be computed with the trapezoids method. Indeed, the singularity of the integrand at $\vartheta=0$ is integrable, as long as the dimensionality is sufficient and the band structure is well-behaved $(k$ linear terms, $k^{3}$-terms, and the anisotropy may play a role here). The RKKY (i.e., intraband) term is omitted in this discussion, as the density of states vanishes if the Fermi energy $E_{F} \rightarrow 0$.

The computations have been performed with an efficient algorithm based on the fast Fourier transform on a $16 \times 16 \times 16$ supercell (16384 cation lattice sites) that also determines the grid density of $k$ and $k^{\prime}$ points employing periodic boundary conditions. For $\mathrm{Cd}_{1-x} \mathrm{Mn}_{x}$ Te and $\mathrm{Hg}_{1-x} \mathrm{Mn}_{x} \mathrm{Te}$, the grids with up to two and eight different $\vartheta$ values have been employed, respectively. Figure 1 demonstrates that the magnitude of $\Theta_{h e}$ in $\mathrm{Hg}_{1-x} \mathrm{Mn}_{x} \mathrm{Te}$ converges with the number of employed $\vartheta$ values. This means that in contrast to the static dielectric function [43. 44, the spin susceptibility, though enhanced, is not singular at $q \rightarrow 0$ in the symmetry-induced zero-gap semiconductors. Aitken's delta-squared process served to accelerate the integration convergence. We have checked that $\Theta_{0}$ calculated by the single integral, as outlined in a preceding paragraph, and after subtracting $J_{0}$, is in excel-
TABLE I: Consecutive nearest-neighbor exchange energies $-\left(J_{i}=J_{i, h h}+J_{i, e e}+J_{i, h e}\right), i=1,2,3,4$ (from the fourth-order perturbation theory) and Curie-Weiss parameter $-\Theta_{0}$ in Kelvins compared to experimental results. Contributions from the superexchange $(h h)$, electron-electron $(e e)$, and interband $(h e)$ terms to $\Theta_{0}$

\begin{tabular}{|c|c|c|c|c|c|c|c|}
\hline & \multicolumn{5}{|c|}{$\mathrm{Cd}_{1-x} \mathrm{Mn}_{x} \mathrm{Te}$} & \multicolumn{2}{|c|}{$\mathrm{Hg}_{1-x} \mathrm{Mn}_{x} \mathrm{Te}$} \\
\hline & \multicolumn{2}{|c|}{ theory } & \multicolumn{3}{|c|}{ expl } & theory & expl \\
\hline$-J_{1}$ & \multicolumn{2}{|c|}{9.77} & \multicolumn{3}{|c|}{$\begin{array}{c}6.3 \pm 0.3[45 \\
6.15 \pm 0.05[47\end{array}$} & 6.46 & $\begin{array}{l}5.1 \pm 0.546 \\
4.3 \pm 0.548\end{array}$ \\
\hline$-J_{2}$ & \multicolumn{2}{|c|}{0.810} & \multicolumn{3}{|c|}{$\begin{array}{c}1.9 \pm 1.145 \\
1.80 \pm 0.05 \text { 47 }\end{array}$} & 0.842 & \\
\hline$-J_{3}$ & \multicolumn{2}{|c|}{0.352} & \multicolumn{3}{|c|}{$\begin{array}{c}0.4 \pm 0.345 \\
1.39 \pm 0.0547\end{array}$} & 0.394 & \\
\hline$-J_{4}$ & \multicolumn{2}{|c|}{0.255} & \multicolumn{3}{|c|}{$0.81 \pm 0.05[4]$} & 0.467 & \\
\hline$-\Theta_{0}$ & \multicolumn{2}{|c|}{801} & \multicolumn{3}{|c|}{$470 \pm 34[25$} & 666 & $\begin{array}{l}500 \pm 1025 \\
660 \pm 8826 \\
\end{array}$ \\
\hline \multicolumn{8}{|c|}{ theory } \\
\hline$\overline{-J}$ & $1, h e$ & -0 . & .396 & $-\Theta_{h h}$ & 772 & -1.583 & 651 \\
\hline$-J$ & $2, h e$ & & 421 & $-\Theta_{e e}$ & 8.9 & 0.492 & 19.0 \\
\hline$-J$ & $3, h e$ & & 131 & $-\Theta_{h e}$ & 20.0 & 0.112 & -3.9 \\
\hline$-J$ & $4, h e$ & & 061 & & & 0.154 & \\
\hline
\end{tabular}
are also shown.

lent numerical agreement (better than $1 \mathrm{~K}$ ) with the value obtained by summing $z_{i} J_{i}, i \geq 1$, obtained by the double BZ integration. Actually, the coupling to the nearest neighbors contributes over $50 \%$ to the value of $\Theta_{0}$.

\section{over 50}

Discussion of theoretical results vis-à-vis experimental data. As expected theoretically for the random distribution of magnetic ions, experimental values of $\Theta_{\mathrm{CW}}$ show linear dependence on $x$ in II-VI DMSs [25, 51]. As shown in Table I, our theory and together with the employed tight-binding parametrization explains the interaction sign but overestimate by $60 \%$ the absolute values of $J_{1}$ and $\Theta_{0}$ in $\mathrm{Cd}_{1-x} \mathrm{Mn}_{x}$ Te and by $30 \%$ in the case of 

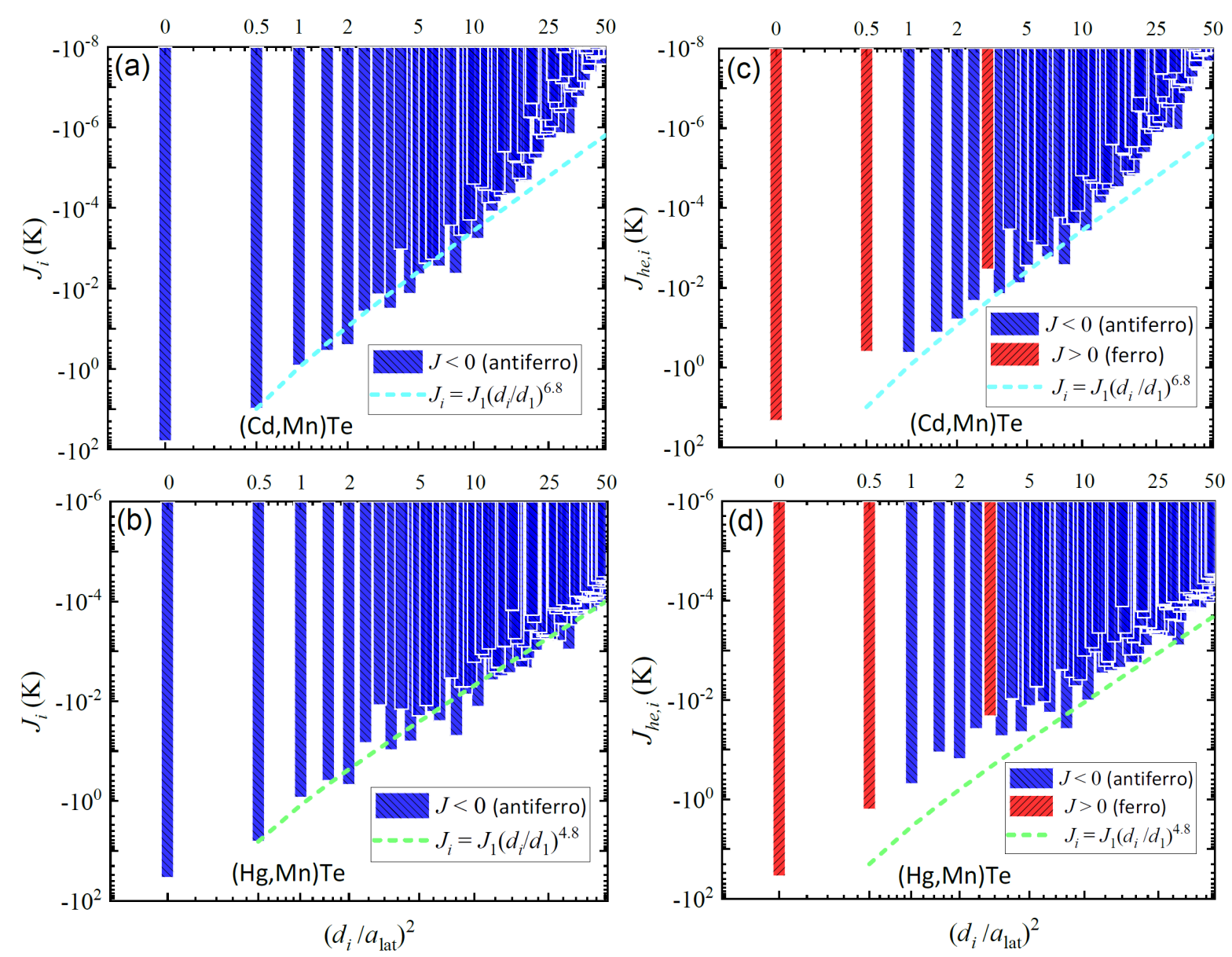

FIG. 2: Computed total exchange energies $J_{i}(\mathrm{a}, \mathrm{b})$ and the interband BR contribution $J_{i, h e}(\mathrm{c}, \mathrm{d})$ for Mn pairs (including the self-interaction values $J_{0}$ ) vs. Mn-Mn distances $d_{i}$ in the unit of the lattice parameter $a_{\text {lat }}$ for $\mathrm{CdTe}$ (a,c) and $\operatorname{HgTe}(\mathrm{b}, \mathrm{d})$. Dashed lines indicate $J_{i} \sim d_{i}^{-n}$ with $n=6.8$ and 4.8, as found experimentally for $\mathrm{Cd}_{1-x} \mathrm{Mn}_{x} \mathrm{Te}$ [49] and $\mathrm{Hg}_{1-x} \mathrm{Mn}_{x} \mathrm{Te}$ [50], respectively.

$\mathrm{Hg}_{1-x} \mathrm{Mn}_{x} \mathrm{Te}$.

Extensive experimental studies of spin-glass freezing temperature $T_{f}$ in wide-gap Mn- and Co-based DMSs, including $\mathrm{Cd}_{1-x} \mathrm{Mn}_{x} \mathrm{Te}$, indicate that $\Theta_{\mathrm{CW}} \gg T_{f} \sim x^{\alpha}$, where $\alpha=2.25 \pm 0.1$ [40, 49, 50, 52]. A scaling argument [49, 53] then implies $J_{i} \sim d_{i}^{-n}$, where $d_{i}$ is the distance between spin pairs and $n=3 \alpha=6.8 \pm 0.3$ [49]. Figure2(a) demonstrates that the dependence of $J_{i}$ on $d_{i}$ obtained here for $\mathrm{Cd}_{1-x} \mathrm{Mn}_{x} \mathrm{Te}$ is in agreement with the experimentally determined power law. However, an exponential decay would describe the computed data over a wider range of $d_{i}$.

Comparing $J_{i, h e}$ values displayed in Figs. 2(c,d) to $J_{l}$ data in Figs.2(a,b), we find that the BR mechanism dominates at large $d$. It decays exponentially with $d$ in the wide-gap $\mathrm{Cd}_{1-x} \mathrm{Mn}_{x} \mathrm{Te}$ but for topological zero-gap $\mathrm{Hg}_{1-x} \mathrm{Mn}_{x} \mathrm{Te}, J_{i, h e}(d)$ shows a power-law dependence, also at large $d$. This behavior accounts for a relatively weak decay of $T_{f}$ with decreasing $x$ in $\mathrm{Hg}_{1-x} \mathrm{Mn}_{x} \mathrm{Te}$ [54], leading to $n=4.8$ [50]. As seen in Fig. 2(b), this value of $n$ is consistent with our theoretical results, though we have to note that a considerable shift of bands with $x$ is expected in topological materials, while our computations have been performed for Mn pairs in HgTe. As shown in Table I, the relevant ferromagnetic and antiferromagnetic contributions $J_{i, h e}, i \geq 1$ actually cancel each other in $\Theta_{h e}$, making the contribution of the BR mechanism to $\Theta_{0}$ negligible in both compounds. This explains why no effect of gap opening on $\Theta_{\mathrm{CW}}$ was found in $\mathrm{Hg}_{1-x-y} \mathrm{Cd}_{y} \mathrm{Mn}_{x} \mathrm{Te}$ [26. At the same time, it is clear from Fig. 1 that the inclusion of the self-interaction term $J_{0, h e}$, would drastically increase the magnitude of $\Theta_{h e}$.

Conclusions and outlook. Our results demonstrate that the interband BR term changes the sign from ferromagnetic to antiferromagnetic as a function of Mn pair distance, with the behavior contradicting the Van Vleck-like approach that predicts only the ferromagnetic coupling [16. Such an alternating sign, reflecting the presence of both ferromagnetic and antiferromagnetic excitations in $\tilde{\chi}(q)$ [55], significantly reduces the role of the interband contribution making the superexchange to determine whether a spin-glass or a ferromagnet becomes the 
magnetic ground state, the case of $\mathrm{Mn}^{2+}$ in II-VI compounds and $\mathrm{Mn}^{3+}$ in GaN, respectively.

There are persisting uncertainties concerning the distribution (random vs. clustering [56]) and the location of transition metal (TM) impurities in the tetradymite lattice (substitutional vs. interstitial positions in the van der Waals gap [57]). Nevertheless, a series of arguments allows extending the conclusion about the dominance of the superexchange to topological tetradymite chalcogenides doped by substitutional V, Cr, or Fe ions, whose magnetism has so far been merely attributed to the Van Vleck mechanism [9, 10]. (i) These impurities appear isoelectronic [9, 10, 27], which means that $d$ orbitals remain fully occupied or empty. Moreover, as in other DMSs, correlations, together with the Jahn-Teller effect and dilution, enhance the $d$ orbital localization further on. Accordingly, the double-exchange scenario, put forward when interpreting $a b$ initio results [28, 30, is not valid. (ii) Another $a b$ initio study reveals the insensitivity of the spin-spin coupling energy of the band inversion [29], the finding contradicting the Van Vleck model. (iii) Recent studies of x-ray magnetic circular dichroism and resonant photoelectron spectroscopy demonstrate similarities of $p$ $d$ hybridization effects in $\mathrm{V}$ - or Cr-doped $\left(\mathrm{Bi}_{x} \mathrm{Sb}_{1-x}\right)_{2} \mathrm{Te}_{3}$ 30] and II-VI DMSs [41, in particular, stronger hybridization of $t_{2 g}$ TM levels compared to the $e_{g}$ case, which implies a similar physics of spin-spin coupling as found in tetrahedrally coordinated DMSs. (iv) As superexchange prevails over the interband Van Vleck mechanism in the zero-gap case, it should dominate even more strongly in the gapped topological systems. (v) From the direct computations for tetrahedral systems carried out here and previously [36, 38, 58, supported by experimental data [40, 49, 50, 59, 60, we know that the superexchange is ferromagnetic for $d^{3}$ and $d^{4}$, whereas it is antiferromagnetic for $d^{5}$ and $d^{6}$ cases. According to experimental results [9, 10, 27] and $a b$ initio studies [28], the same sequence occurs in tetradymite topological insulators, except for the Mn case, as Mn acts as an acceptor 61, so that the RKKY interaction accompanies the antiferromagnetic superexchange, such as (Ga,Mn)As [6, 62. Altogether, these arguments indicate that the TM charge state and coordination, more than a topological class, govern the magnetic properties of DMSs.

Acknowledgments. The work is supported by the Foundation for Polish Science through the International Research Agendas program co-financed by the European Union within the Smart Growth Operational Programme. One of us (JAM) acknowledges support of the National Science Centre (Poland) under the grant UMO-218/31/BST3/03758. We acknowledge the access to the computing facilities of the Interdisciplinary Center of Modeling at the University of Warsaw, Grant No. G6812 . 


\section{Supplemental Material}

\section{OVERVIEW OF VARIOUS EXCHANGE MECHANISMS}

Since the exchange part of the Coulomb energy decays exponentially with the distance between magnetic ions, the spin-spin interaction in transition-metal compounds is dominated by indirect coupling via non-magnetic states residing in-between localized spins [1, 41. Quite generally, the presence of $d$ orbitals brought about by transition metals leads to additional delocalization of valence electrons, i.e., to the lowering of their quantum mechanical kinetic energy, as illustrated in Fig. S1 63. The effect is spin dependent as, according to the Hund's rule and due to the on-site Hubbard energy $U, d$-orbitals are spin-polarized in transition metals.

Recently, we proposed a formal theory of the indirect spin-dependent interaction resulting from hybridization between $d$-orbitals of magnetic ions and $s p$ band states [21. That theory, within the second order in the hybridization energy $V_{\text {hyb }}$ provides exchange integrals describing Kondo-like coupling between localized spins and band states $J_{s p-d}$ [21, 22, 41], whereas the fourth order in $V_{\text {hyb }}$ allows to obtain the exchange tensor of the interaction between pairs of the localized spins [21, 23, 41]. This procedure encompasses on equal footing various spin-spin exchange mechanisms, including superexchange, Ruderman-Kittel-Kasuya-Yosida (RKKY) and Bloembergen-Rowland-Van Vleck (BR-VV) interactions, the latter two usually described within the second order perturbation theory in $J_{s p-d}$, as discussed in the next section. We recall that these two mechanisms of coupling between localized spins can be viewed as mediated by spin-polarization of band, carriers and/or occupied bands, in an analogy to intraband and interband dielectric polarizations. However, in the absence of band carriers, the dominant interaction is the superexchange described in the fourth order in $V_{\text {hyb }}$ by a diagram depicted in Fig. S2(a) 41. As shown in the main body of our paper, if the self-interaction term is treated correctly, i.e., omitted when considering pairwise interactions, the superexchange [Fig. S2(a)] dominates over the interband BR-VV contribution [Fig. S2(b)], even in the case of zero-gap $\operatorname{Hg}_{1-x} \mathrm{Mn}_{x} \mathrm{Te}_{\text {. }}$

\section{FOURTH-ORDER VS. SECOND-ORDER PERTURBATION THEORY}

Typically, as mentioned in the previous Section, the RKKY interaction is assumed to originate from a Kondo-like contact interaction $\hat{\mathcal{H}}_{p d}$,

$$
\hat{\mathcal{H}}_{p d}=-\frac{1}{\mathcal{V}}\left(\hat{J}_{p d}(\mathbf{r}) \hat{\mathbf{s}}\right) \cdot \hat{\mathbf{S}}_{i}
$$

where $\hat{\mathbf{s}}$ and $\hat{\mathbf{S}}_{i}$ are the band electron's and impurity's spin operators, respectively, whereas the usual exchange integral $J_{p d}$ has been replaced with a Hermitian operator $\hat{J}_{p d}(\hat{\mathbf{r}})$. For an Anderson impurity, $\hat{\mathcal{H}}_{p d}$ is considered a second-order effective Hamiltonian that can be obtained via the Schrieffer-Wolff transformation [64]. We imagine that this operator is a product, $\hat{J}_{p d}(\hat{\mathbf{r}}) \equiv J_{p d} \hat{P}(\hat{\mathbf{r}})$, of a constant $J_{p d}$ and a Hermitian operator $\hat{P}(\hat{\mathbf{r}})$, the latter restricting the interaction to the tight-binding orbitals of appropriate symmetry [namely, the $p$ orbitals of the anion; in the effective-mass

(a) without $p-d$ hybridization

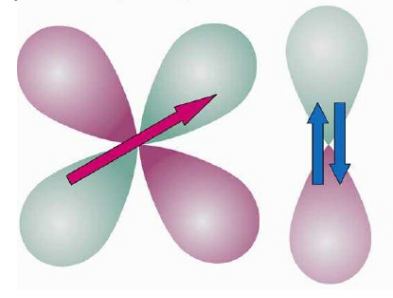

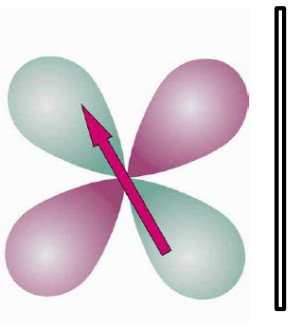

(b) with $p-d$ hybridization

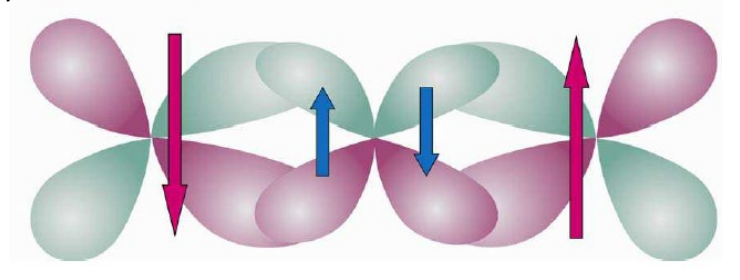

FIG. S1: Schematic illustration of open $d^{5}$ orbitals at the transition metal cations and entirely occupied $p$ orbitals at the anion without (a) and with (b) $p$ - $d$ hybridization. A spin-dependent shift of the orbitals (described by the fourth order perturbation theory), occurring for an antiferromagnetic arrangement of Mn spins (red arrows) shown in (b), leads to lowering of the quantum kinetic energy associated with delocalization of electrons over the $p$ and $d$ orbitals.

Note that virtual $p$ - $d$ transitions occurs only for antiparallel orientation of Mn (red arrows) and anion spins (blue arrows). 

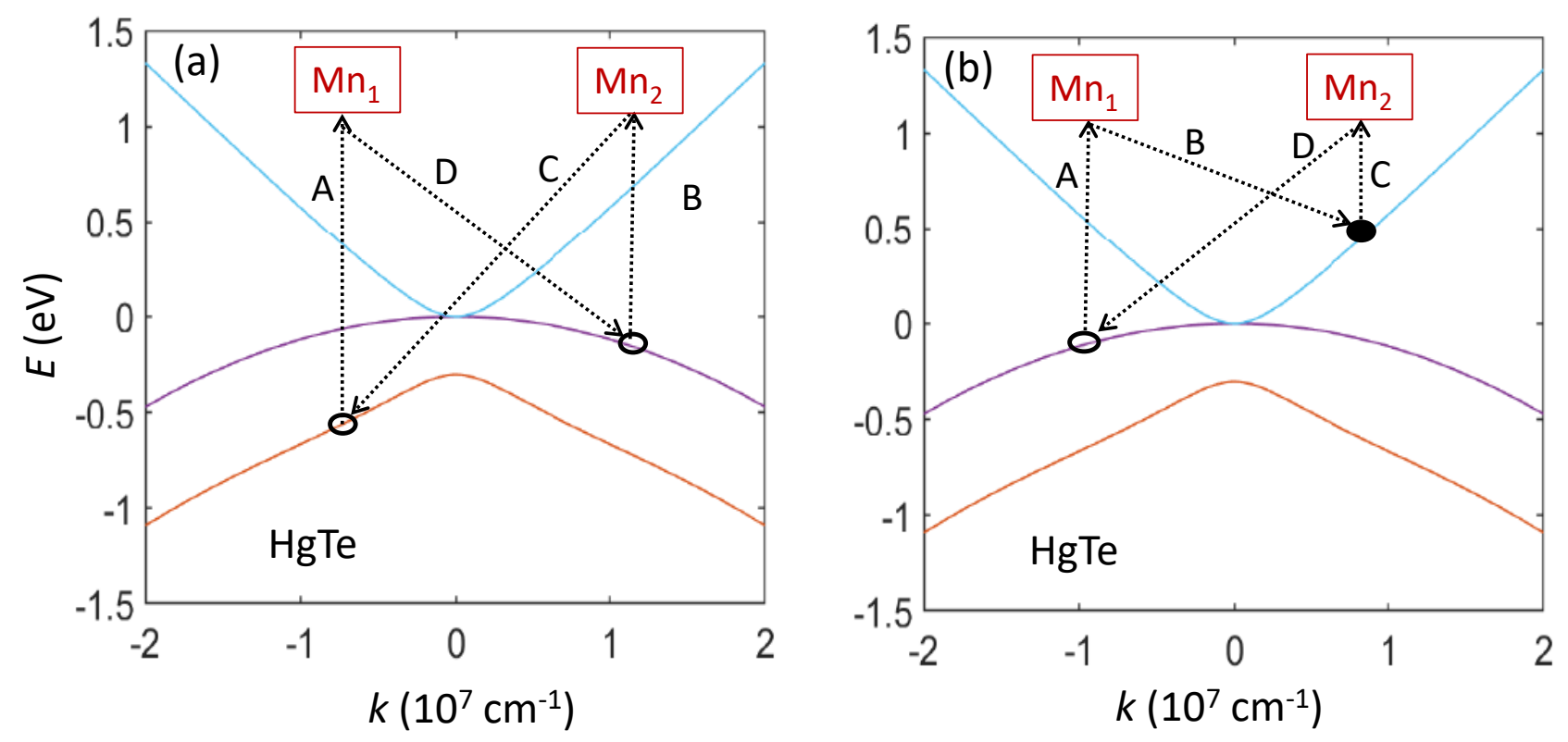

FIG. S2: Examples of four virtual transitions allowed by hybridization between band states and $d$ orbitals of two Mn ions (labeled 1 and 2) in zero-gap HgTe. These processes account for (a) superexchange (the $h h$ contribution) and (b) Bloembergen-Rowland-Van Vleck coupling (the $e h$ contribution).

approximation $\hat{P}$ includes a Dirac delta at the impurity position $\left.\mathbf{R}_{i}, \delta\left(\hat{\mathbf{r}}-\mathbf{R}_{i}\right)\right]$. The Hamiltonian for two impurities $(i, j)$ reads

$$
\hat{\mathcal{H}}_{\mathrm{eff}(2)}=-\frac{1}{\mathcal{V}} \hat{J}_{p d}(\hat{\mathbf{r}}) \hat{\mathbf{s}} \cdot\left(\hat{\mathbf{S}}_{i}+\hat{\mathbf{S}}_{j}\right) .
$$

By applying a second order thermodynamic perturbation theory we obtain the Landau free energy $\Omega$ for the gas of band electrons,

$$
\Omega^{(2 \times 2)}=\sum_{k, k^{\prime}} \frac{f\left(E_{k}\right)}{E_{k}-E_{k^{\prime}}}\left\langle k\left|\hat{H}_{\mathrm{eff}(2)}\right| k^{\prime}\right\rangle\left\langle k^{\prime}\left|\hat{H}_{\mathrm{eff}(2)}\right| k\right\rangle
$$

$(2 \times 2$ stands for the second order perturbation theory on the second order effective Hamiltonian, yielding effectively the fourth order). It includes an interaction term,

$$
\Omega_{i, j}^{(2 \times 2)}=\sum_{\alpha \beta} \frac{2 S_{i \alpha} S_{j \beta}}{\mathcal{V}^{2}} \sum_{k, k^{\prime}} \frac{f\left(E_{k}\right)}{E_{k}-E_{k^{\prime}}}\left\langle k^{\prime}\left|\hat{J}_{p d}(\mathbf{r}) \hat{s}_{\alpha}\right| k\right\rangle\left\langle k\left|\hat{J}_{p d}(\hat{\mathbf{r}}) \hat{s}_{\beta}\right| k^{\prime}\right\rangle
$$

where the impurities are assumed to be in spin-coherent states, $\left\langle\hat{S}_{i \alpha}\right\rangle=S_{i \alpha}$. Therefore the low-temperature interaction constants, $J_{i j, \alpha \beta}^{(2 \times 2)}$, are given by

$$
J_{i j, \alpha \beta}^{(2 \times 2)}=-\frac{J_{p d}^{2}}{\mathcal{V}^{2}} \sum_{k, k^{\prime}} \frac{f\left(E_{k}\right)}{E_{k}-E_{k^{\prime}}}\left\langle k^{\prime}\left|\hat{P} \hat{s}_{\alpha}\right| k\right\rangle\left\langle k\left|\hat{P} \hat{s}_{\beta}\right| k^{\prime}\right\rangle .
$$

Since (according to Schrieffer and Wolff [64])

$$
J_{p d}=\frac{1}{S} \frac{U}{\left(E_{d}-E_{F}\right)\left(E_{d}+U-E_{F}\right)}\left|\tilde{V}_{k_{F}}\right|^{2},
$$

we obtain

$$
J_{i j, \alpha \beta}^{(2 \times 2)}=-\frac{1}{2(2 S)^{2}} \frac{U^{2}}{\left(E_{d}-E_{F}\right)^{2}\left(E_{d}+U-E_{F}\right)^{2}} \sum_{k, k^{\prime}} \frac{f\left(E_{k}\right)-f\left(E_{k^{\prime}}\right)}{E_{k}-E_{k^{\prime}}}\left\langle k^{\prime}\left|\hat{Q} \hat{\sigma}_{\alpha}\right| k\right\rangle\left\langle k\left|\hat{Q} \hat{\sigma}_{\beta}\right| k^{\prime}\right\rangle,
$$


with $\hat{Q}=\frac{1}{\mathcal{V}}\left|\tilde{V}_{k_{F}}\right|^{2} \hat{P}$. Furthermore, by writing

$$
\hat{Q}=\hat{V}_{\text {hyb }}^{\dagger}\left(\sum_{m ; a=\uparrow, \downarrow}\left|d_{i} m a\right\rangle\left\langle d_{i} m a\right|\right) \hat{V}_{\text {hyb }}
$$

and assuming that the hybridization is spin-independent:

$$
\hat{Q} \hat{\sigma}_{\alpha}=\hat{V}_{\mathrm{hyb}}^{\dagger}\left(\sum_{m ; a, b=\uparrow, \downarrow}\left|d_{i} m a\right\rangle\left\langle a\left|\sigma_{\alpha}\right| b\right\rangle\left\langle d_{i} m b\right|\right) \hat{V}_{\mathrm{hyb}},
$$

we reproduce some of the he terms (those with the singular denominator), neglecting the dependence on the band energies $\left(E_{k}, E_{k^{\prime}}\right)$.

The remaining he terms (those proportional to $2 / U$ ) can be derived from the second-order quasi-degenerate perturbation theory [65] as follows. Consider the six-dimensional Hilbert space for two Anderson impurities occupied by two electrons. The ground state corresponds to a single occupation of each impurity and is four-fold spin-degenerate, the remaining two states are higher in energy by $U$. The $2 / U$ terms appear on restricting the Hamiltonian to the four-dimensional ground state via application of the Winkler's perturbation theory (in the second order).

\section{BLOEMBERGEN-ROWLAND MECHANISM: INTEGRATION CONVERGENCE}

In this Section, the BR-VV interaction in a zero-gap semiconductor $\left(E_{g}=0\right)$ with spherical, parabolic bands is considered in the case of accidental degeneracy, $E_{c(v)}=+(-) \hbar^{2} k^{2} / 2 m_{c(v)}$. The double reciprocal-space integral that arises is not absolutely convergent. It may be truncated at a finite momentum [17, 20] or understood in the improper

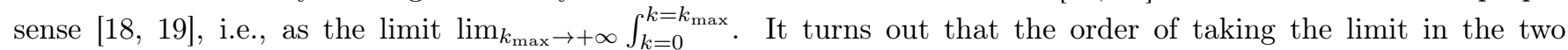
integrals (if the integral is calculated as an iterated one) may affect the result of the double-integration procedure, as evidenced by the violation of the expected symmetry between the valence and conduction bands $\left(m_{c} \leftrightarrow m_{v}\right)$ [18]. In the extreme case, even the condition $J_{0}+\sum_{i>1} z_{i} J_{i}>0$, may be violated. This difficulties appear as unavoidable and make estimation of the interaction within a simple model rather complex, as elaborated previously. With the improper sense in place and the Fermi level in the conduction band $\left(\hbar k_{F}=\sqrt{2 m_{c} E_{F}}\right)$, the Hamiltonian for a system of two localized spins assumes the form:

$$
\begin{aligned}
& \mathcal{H}\left(R_{i j}\right)=-\frac{2 J_{c v}^{2}}{(2 \pi)^{3} \hbar^{2}} \frac{\left(m_{c} m_{v}\right)^{3 / 2}}{\left(m_{c}+m_{v}\right)^{2}} \frac{\exp \left(-\sqrt{m_{v} / m_{c}} k_{F} R_{i j}\right)}{R_{i j}^{4}} \times \\
& \times\left[\left(2+\frac{m_{c}+m_{v}}{\sqrt{m_{c} m_{v}}} k_{F} R_{i j}\right) \cos \left(k_{F} R_{i j}\right)+\left(\frac{m_{v}-m_{c}}{\sqrt{m_{c} m_{v}}}+\frac{m_{c}+m_{v}}{m_{c}} k_{F} R_{i j}\right) \sin \left(k_{F} R_{i j}\right)\right] \mathbf{S}_{i} \cdot \mathbf{S}_{j},
\end{aligned}
$$

where $J_{c v}$ is an interband $s p$ - $d$ exchange integral. In contrast to Ref.17, where a hard momentum cut-off $k_{t}$ was introduced, the expression is not integrable at $R_{i j}=0$, and as such, is significantly different from Eq. 32 in Ref.17. Assuming a real-space cut-off at $R_{\min }$,

$$
\begin{aligned}
& \Theta_{h e}=N_{0}\left(\frac{J_{c v}}{\pi \hbar}\right)^{2} \frac{m_{c} m_{v}}{\left(m_{c}+m_{v}\right)^{2}} \frac{\exp \left(-\sqrt{m_{v} / m_{c}} k_{F} R_{\min }\right)}{R_{\min }} \times \\
& \times\left[2 \sqrt{m_{c} m_{v}} \cos \left(k_{F} R_{\min }\right)-\left(m_{c}-m_{v}\right) \sin \left(k_{F} R_{\min }\right)\right] \frac{S(S+1)}{3} .
\end{aligned}
$$

The oscillations as a function of $k_{F} R_{\min }$ are damped exponentially with $\alpha=\sqrt{m_{v} / m_{c}}$ and shifted in phase by $\phi=\arctan \left[\left(m_{c}-m_{v}\right) / 2 \sqrt{m_{c} m_{v}}\right]$. Taking, for instance, $R_{\min }=\left(4 \pi N_{0}\right)^{-1 / 3}$, and parameters for intrinsic HgTe, $k_{F}=0, a_{\text {lat }}=0.646 \mathrm{~nm}, N_{0} J_{c v}=-0.6 \mathrm{eV} ; m_{c}=0.3 m_{0}$, and $m_{v}=0.45 m_{0}$, we obtain ferromagnetic $\Theta_{h e}=85 \mathrm{~K}$.

An alternative procedure is proposed here which amounts to regularizing the momentum integrals by introducing under the integral an additional weight, $d k \rightarrow \exp (-a k) d k, a \rightarrow 0^{+}$. One proceeds with integration over one of the momenta $(k)$ and takes the limit $a \rightarrow 0^{+}$. Then, an additional factor appears which exponentially divergent and must be suppressed by $d k^{\prime} \rightarrow \exp \left(-a^{\prime} k^{\prime}\right) d k^{\prime}$ with sufficiently large $a^{\prime}$. However, the result of the second integration is analytic with respect to $a^{\prime}$ and can be continued to $a^{\prime}=0^{+}$, where its value vanishes as $a^{\prime} \rightarrow 0^{+}$. Furthermore, although the exchange constant diverges as $1 / a^{\prime}$ if integrated over the real space $\left(R_{i j}\right)$, the real space integral vanishes 
again if the vicinity of $R_{i j}=0$ is omitted from the integration. One concludes that this simple model predicts $\Theta_{h e}=0$, and the interaction vanishes except for $R_{i j} \rightarrow 0$. In practice, values of $a$ and $a^{\prime}$ of the order of the lattice constant are appropriate, and result in a real-space-distance dependence of the exchange constant similar to that presented in Fig. 2 in the main text.

[1] Robert M. White, Quantum Theory of Magnetism (Springer-Verlag, Berlin, 2007) pp. 1-213.

[2] C. Zener, "Interaction between the d shells in the transition metals," Phys. Rev. 81, 440 (1951).

[3] T. Dietl and J. Spałek, "Effect of fluctuations of magnetization on the bound magnetic polaron: Comparison with experiment," Phys. Rev. Lett. 48, 355-358 (1982).

[4] T. Dietl, "Spin dynamics of a confined electron interacting with magnetic or nuclear spins: A semiclassical approach," Phys. Rev. B 91, 125204 (2015).

[5] T. Dietl, H. Ohno, F. Matsukura, J. Cibert, and D. Ferrand, "Zener model description of ferromagnetism in zinc-blende magnetic semiconductors," Science 287, 1019-1022 (2000)

[6] T. Dietl and H. Ohno, "Dilute ferromagnetic semiconductors: Physics and spintronic structures," Rev. Mod. Phys. 86, 187-251 (2014)

[7] T. Jungwirth, J. Wunderlich, V. Novák, K. Olejník, B. L. Gallagher, R. P. Campion, K. W. Edmonds, A. W. Rushforth, A. J. Ferguson, and P. Němec, "Spin-dependent phenomena and device concepts explored in (Ga,Mn)As," Rev. Mod. Phys. 86, 855-896 (2014).

[8] T. Dietl, A. Haury, and Y. Merle d'Aubigne, "Free carrier-induced ferromagnetism in structures of diluted magnetic semiconductors," Phys. Rev. B 55, R3347 (1997)

[9] He Ke, Yayu Wang, and Qi-Kun Xue, "Topological materials: quantum anomalous Hall system," Annu. Rev. Cond. Mat. Phys. 9, 329-344 (2018).

[10] Y. Tokura, K. Yasuda, and A. Tsukazaki, "Magnetic topological insulators," Nat. Rev. Phys. 1, 126-143 (2019)

[11] Cui-Zu Chang, Jinsong Zhang, Xiao Feng, Jie Shen, Zuocheng Zhang, Minghua Guo, Kang Li, Yunbo Ou, Pang Wei, Li-Li Wang, Zhong-Qing Ji, Yang Feng, Shuaihua Ji, Xi Chen, Jinfeng Jia, Xi Dai, Zhong Fang, ShouCheng Zhang, Ke He, Yayu Wang, Li Lu, Xu-Cun Ma, and Qi-Kun Xue, "Experimental observation of the quantum anomalous Hall effect in a magnetic topological insulator," Science 340, 167-170 (2013).

[12] Di Xiao, Jue Jiang, Jae-Ho Shin, Wenbo Wang, Fei Wang, Yi-Fan Zhao, Chaoxing Liu, Weida Wu, Moses H. W. Chan, N. Samarth, and Cui-Zu Chang, "Realization of the axion insulator state in quantum anomalous Hall sandwich heterostructures," Phys. Rev. Lett. 120, 056801 (2018)

[13 Yabin Fan, P. Upadhyaya, Xufeng Kou, Murong Lang, So Takei, Zhenxing Wang, Jianshi Tang, Liang He, LiTe Chang, M. Montazeri, Guoqiang Yu, Wanjun Jiang, Tianxiao Nie, R. N. Schwartz, Y Tserkovnyak, and Kang L. Wang, "Magnetization switching through giant spin-orbit torque in a magnetically doped topological insulator heterostructure," Nat. Mater. 13, 699-704
(2014)

[14] Qing Lin He, Lei Pan, A. L. Stern, E. C. Burks, Xiaoyu Che, Gen Yin, Jing Wang, Biao Lian, Quan Zhou, Eun Sang Choi, K. Murata, Xufeng Kou, Zhijie Chen, Tianxiao Nie, Qiming Shao, Yabin Fan, Shou-Cheng Zhang, Kai Liu, Jing Xia, and Kang L. Wang, "Chiral Majorana fermion modes in a quantum anomalous Hall insulator-superconductor structure," Science 357, 294-299 (2017)

[15] M. Kayyalha, Di Xiao, Ruoxi Zhang, Jaeho Shin, Jue Jiang, Fei Wang, Yi-Fan Zhao, Run Xiao, Ling Zhang, K. M. Fijalkowski, Pankaj Mandal, M. Winnerlein, C. Gould, Qi Li, L. W. Molenkamp, Moses H. W. Chan, N. Samarth, and Cui-Zu Chang, "Absence of evidence for chiral Majorana modes in quantum anomalous Hallsuperconductor devices," Science 367, 64-67 (2020).

[16] Rui Yu, Wei Zhang, Hai-Jun Zhang, Shou-Cheng Zhang, $\mathrm{Xi}$ Dai, and Zhong Fang, "Quantized anomalous Hall effect in magnetic topological insulators," Science 329, 61-64 (2010), see Eq. (S1) in Supporting Online Material.

[17] N. Bloembergen and T. J. Rowland, "Nuclear spin exchange in solids: $\mathrm{Tl}^{203}$ and $\mathrm{Tl}^{205}$ magnetic resonance in thallium and thallic oxide," Phys. Rev. 97, 1679-1698 (1955)

[18] G. Bastard and C. Lewiner, "Indirect-exchange interactions in zero-gap semiconductors," Phys. Rev. B 20, 4256-4267 (1979)

[19] C. Lewiner and G. Bastard, "Indirect exchange interaction in extremely non-parabolic zero-gap semiconductors," J. Phys. C: Solid State Physics 13, 2347-2360 (1980)

[20| Ven-Chung Lee, "Indirect exchange interaction in the zero-gap semiconductor $\mathrm{Hg}_{1-\mathrm{x}} \mathrm{Mn}_{\mathrm{x}}$ Te," Phys. Rev. B 37, 8849-8854 (1988)

[21] C. Sliwa and T. Dietl, "Thermodynamic perturbation theory for noninteracting quantum particles with application to spin-spin interactions in solids," Phys. Rev. B 98, 035105 (2018).

[22] C. Autieri, C. Sliwa, R. Islam, G. Cuono, and T. Dietl, "Momentum-resolved spin splitting in Mn-doped trivial CdTe and topological HgTe semiconductors," Phys. Rev. B 103, 115209 (2021)

[23] B. E. Larson, K. C. Hass, H. Ehrenreich, and A. E. Carlsson, "Theory of exchange interactions and chemical trends in diluted magnetic semiconductors," Phys. Rev. B 37, 4137-4154 (1988)

[24] A. Savoyant, S. D’Ambrosio, R. O. Kuzian, A. M. Daré, and A. Stepanov, "Exchange integrals in Mn- and Codoped II-VI semiconductors," Phys. Rev. B 90, 075205 (2014)

[25] J. Spałek, A. Lewicki, Z. Tarnawski, J. K. Furdyna, R. R. Galazka, and Z. Obuszko, "Magnetic susceptibility of semimagnetic semiconductors: The high-temperature 
regime and the role of superexchange," Phys. Rev. B 33, 3407-3418 (1986)

[26] A. Lewicki, J. Spałek, J. K. Furdyna, and R. R. Gałazka, "Magnetic susceptibility of diluted magnetic (semimagnetic) semiconductors: Further evidence for superexchange," Phys. Rev. B 37, 1860-1863 (1988).

[27] Y. Satake, J. Shiogai, G. P. Mazur, S. Kimura, S. Awaji, K. Fujiwara, T. Nojima, K. Nomura, S. Souma, T. Sato, T. Dietl, and A. Tsukazaki, "Magnetic-field-induced topological phase transition in Fe-doped (Bi, $\mathrm{Sb})_{2} \mathrm{Se}_{3}$ heterostructures," Phys. Rev. Materials 4, 044202 (2020).

[28] M. G. Vergniory, M. M. Otrokov, D. Thonig, M. Hoffmann, I. V. Maznichenko, M. Geilhufe, X. Zubizarreta, S. Ostanin, A. Marmodoro, J. Henk, W. Hergert, I. Mertig, E. V. Chulkov, and A. Ernst, "Exchange interaction and its tuning in magnetic binary chalcogenides," Phys. Rev. B 89, 165202 (2014).

[29] Jeongwoo Kim, Seung-Hoon Jhi, A. H. MacDonald, and Ruqian $\mathrm{Wu}$, "Ordering mechanism and quantum anomalous Hall effect of magnetically doped topological insulators," Phys. Rev. B 96, 140410(R) (2017).

[30] T. R. F. Peixoto, H. Bentmann, P. Rüßmann, A.-V. Tcakaev, M. Winnerlein, S. Schreyeck, S. Schatz, R. C. Vidal, F. Stier, V. Zabolotnyy, R. J. Green, Chul Hee Min, C. I. Fornari, Maaß H., H. B. Vasili, P. Gargiani, M. Valvidares, A. Barla, J. Buck, M. Hoesch, F. Diekmann, S. Rohlf, M. Kalläne, K. Rossnagel, Ch. Gould, K. Brunner, S. Blügel, V. Hinkov, L. W. Molenkamp, and F. Reinert, "Non-local effect of impurity states on the exchange coupling mechanism in magnetic topological insulators," npj Quantum Materials 5, 87 (2020).

[31] T. Dietl, "(Diluted) Magnetic Semiconductors," in Handbook of Semiconductors, Vol. 3B, edited by S. Mahajan (North Holland, Amsterdam, 1994) p. 1270.

[32] T. Dietl, H. Ohno, and F. Matsukura, "Hole-mediated ferromagnetism in tetrahedrally coordinated semiconductors," Phys. Rev. B 63, 195205 (2001).

[33] D. Ferrand, J. Cibert, A. Wasiela, C. Bourgognon, S. Tatarenko, G. Fishman, T. Andrearczyk, J. Jaroszynski, S. Kolesnik, T. Dietl, B. Barbara, and D. Dufeu, "Carrier-induced ferromagnetism in $p-\mathrm{Zn}_{1-x} \mathrm{Mn}_{x} \mathrm{Te}$," Phys. Rev. B 63, 085201 (2001).

[34] J. Ginter, J. Kossut, and L. Swierkowski, "Indirect exchange interaction via electrons in spin-orbit coupled bands in semiconductors," phys. stat. solidi (b) 96, 735744 (1979)

[35] J. Blinowski and P. Kacman, "Superexchange in $A^{\mathrm{II}} B^{\mathrm{VI}}$ diluted magnetic semiconductors with $\mathrm{Mn}, \mathrm{Fe}$ and Co ions," Acta Phys. Pol. A 87, 539-542 (1995).

[36] J. Blinowski, P. Kacman, and J. A. Majewski, "Ferromagnetic superexchange in Cr-based diluted magnetic semiconductors," Phys. Rev. B 53, 9524 (1996).

[38]

[38] C. Simserides, J. A. Majewski, K. N. Trohidou, and T. Dietl, "Theory of ferromagnetism driven by superexchange in dilute magnetic semi-conductors," EPJ Web of Conferences 75, 01003 (2014).

[39] M. Sawicki, T. Devillers, S. Gałęski, C. Simserides, S. Dobkowska, B. Faina, A. Grois, A. Navarro-Quezada, K. N. Trohidou, J. A. Majewski, T. Dietl, and A. Bonanni, "Origin of low-temperature magnetic ordering in $\mathrm{Ga}_{1-x} \mathrm{Mn}_{x} \mathrm{~N}$," Phys. Rev. B 85, 205204 (2012).

[40] S. Stefanowicz, G. Kunert, C. Simserides, J. A. Majewski, W. Stefanowicz, C. Kruse, S. Figge, Tian Li,
R. Jakieła, K. N. Trohidou, A. Bonanni, D. Hommel, M. Sawicki, and T. Dietl, "Phase diagram and critical behavior of a random ferromagnet $\mathrm{Ga}_{1-x} \mathrm{Mn}_{x} \mathrm{~N}$," Phys. Rev. B 88, 081201(R) (2013).

[41] P. Kacman, "Spin interactions in diluted magnetic semiconductors and magnetic semiconductor structures," Semicon. Sci. Technol. 16, R25-R39 (2001)

[42] S. Froyen, "Brillouin-zone integration by Fourier quadrature: Special points for superlattice and supercell calculations," Phys. Rev. B 39, 3168-3172 (1989).

[43] L. Liu and E. Tosatti, "Dielectric constant and mobility of a doped zero-gap semiconductor," Phys. Rev. Lett. 23, 772-774 (1969)

[44] J. G. Broerman, "Anomalous mobility and dielectric singularity of $\alpha$-Sn," Phys. Rev. Lett. 24, 450-451 (1970).

[45] B. E. Larson, K. C. Hass, and R. L. Aggarwal, "Effects of internal exchange fields on magnetization steps in diluted magnetic semiconductors," Phys. Rev. B 33, 1789-1796 (1986)

[46] R. R. Galazka, W. Dobrowolski, J. P. Lascaray, M. Nawrocki, A. Bruno, J. M. Broto, and J. C. Ousset, "Nearest neighbor exchange constants in $\mathrm{Hg}_{1-x} \mathrm{Mn}_{x} \mathrm{Te}$, $\mathrm{Hg}_{1-x} \mathrm{Mn}_{x}$ Se and other semimagnetic semiconductors," J. Magn. Magn. Mater. 72, 174-180 (1988)

[47] R. V. Cherbunin, V. M. Litviak, I. I. Ryzhov, A. V. Koudinov, S. Elsässer, A. Knapp, T. Kiessling, J. Geurts, S. Chusnutdinow, T. Wojtowicz, and G. Karczewski, "High-resolution resonance spin-flip Raman spectroscopy of pairs of manganese ions in a CdTe quantum well," Phys. Rev. B 101, 241301(R) (2020).

[48] J. P. Lascaray, A. Bruno, J. C. Ousset, H. Rakoto, J. M. Broto, and S. Askenazy, "Quasi-static pulsed high magnetic field used to study magnetic properties of semimagnetic semiconductors," Physica B 155, 353-356 (1989)

[49] A. Twardowski, H. J. M. Swagten, W. J. M. de Jonge, and M. Demianiuk, "Magnetic behavior of the diluted magnetic semiconductor $\mathrm{Zn}_{1-\mathrm{x}} \mathrm{Mn}_{\mathrm{x}}$ Se," Phys. Rev. B 36, 7013-7023 (1987).

[50] R. R. Gałązka, "Influence of electron subsystem on magnetic properties of semimagnetic semiconductors," J. Magn. Magn. Mater. 140-144, 13-16 (1995).

[51] M. Sawicki, E. Guziewicz, M. I. Łukasiewicz, O. Proselkov, I. A. Kowalik, W. Lisowski, P. Dluzewski, A. Wittlin, M. Jaworski, A. Wolska, W. Paszkowicz, R. Jakiela, B. S. Witkowski, L. Wachnicki, M. T. Klepka, F. J. Luque, D. Arvanitis, J. W. Sobczak, M. Krawczyk, A. Jablonski, W. Stefanowicz, D. Sztenkiel, M. Godlewski, and T. Dietl, "Homogeneous and heterogeneous magnetism in ( $\mathrm{Zn}, \mathrm{Co}) \mathrm{O}$ : From a random antiferromagnet to a dipolar superferromagnet by changing the growth temperature," Phys. Rev. B 88, 085204 (2013)

[52] R. R. Galazka, S. Nagata, and P. H. Keesom, "Paramagnetic-spin-glass-antiferromagnetic phase transitions in $\mathrm{Cd}_{1-x} \mathrm{Mn}_{x}$ Te from specific heat and magnetic susceptibility measurements," Phys. Rev. B 22, 3344 (1980)

[53] D. A. Smith, "The zero-frequency susceptibility of spin glasses and micromagnets," J. Phys. F 5, 2148-2167 (1975)

[54] A. Mycielski, C. Rigaux, M. Menant, T. Dietl, and M. Otto, "Spin glass phase transition in $\mathrm{Hg}_{1-k} \mathrm{Mn}_{k} \mathrm{Te}$ semimagnetic semiconductors," Solid State Commun. 50, $257-260(1984)$ 
[55] G. Bednik, "Antiferromagnetism and spin density waves in three-dimensional Dirac metals," Phys. Rev. B 102, 125119 (2020)

[56] Cui-Zu Chang, Peizhe Tang, Yi-Lin Wang, Xiao Feng, Kang Li, Zuocheng Zhang, Yayu Wang, Li-Li Wang, Xi Chen, Chaoxing Liu, Wenhui Duan, Ke He, Xu-Cun Ma, and Qi-Kun Xue, "Chemical-potential-dependent gap opening at the dirac surface states of $\mathrm{Bi}_{2} \mathrm{Se}_{3}$ induced by aggregated substitutional Cr atoms," Phys. Rev. Lett. 112, 056801 (2014).

[57] J. Růžička, O. Caha, V. Holý, H. Steiner, V. Volobuiev, A. Ney, G. Bauer, T. Duchoň, K. Veltruská, I. Khalakhan, V. Matolín, E. F. Schwier, .H Iwasawa, K. Shimada, and G. Springholz, "Structural and electronic properties of manganese-doped $\mathrm{Bi}_{2} \mathrm{Te}_{3}$ epitaxial layers," New J. Phys. 17, 013028 (2015)

[58] J. Blinowski, P. Kacman, and J. A. Majewski, "Superexchange in diluted magnetic semiconductors," Mater. Sci. Forum 182-184, 779-782 (1995)

[59] A. Twardowski, "Magnetic properties of Fe-based diluted magnetic semiconductors," J. Appl. Phys. 67, 5108-5113 (1990)

[60] R. Watanabe, R. Yoshimi, M. Kawamura, M. Mogi, A. Tsukazaki, X. Z. Yu, K. Nakajima, K. S. Takahashi, M. Kawasaki, and Y. Tokura, "Quantum anomalous
Hall effect driven by magnetic proximity coupling in alltelluride based heterostructure," Appl. Phys. Lett. 115, 102403 (2019)

[61] Y. S. Hor, P. Roushan, H. Beidenkopf, J. Seo, D. Qu, J. G. Checkelsky, L. A. Wray, D. Hsieh, Y. Xia, S.Y. Xu, D. Qian, M. Z. Hasan, N. P. Ong, A. Yazdani, and R. J. Cava, "Development of ferromagnetism in the doped topological insulator $\mathrm{Bi}_{2-x} \mathrm{Mn}_{x} \mathrm{Te}_{3}$," Phys. Rev. B 81, 195203 (2010)

[62] K. Sato, L. Bergqvist, J. Kudrnovský, P. H. Dederichs, O. Eriksson, I. Turek, B. Sanyal, G. Bouzerar, H. Katayama-Yoshida, V. A. Dinh, T. Fukushima, H. Kizaki, and R. Zeller, "First-principles theory of dilute magnetic semiconductors," Rev. Mod. Phys. 82, 1633-1690 (2010)

[63] A. Bonanni and T. Dietl, "A story of high-temperature ferromagnetism in semiconductors," Chem. Soc. Rev. 39, 528-539 (2010)

[64] J. R. Schrieffer and P. A. Wolff, "Relation between the Anderson and Kondo hamiltonians," Phys. Rev. 149, 491 (1966).

[65] R. Winkler, Spin-Orbit Coupling Effects in TwoDimensional Electron and Hole Systems Springer Tracts in Modern Physics, Vol. 191 (Springer, Berlin, Heidelberg, 2003) 